\title{
Measurement of the cross section for open-beauty production in photon-photon collisions at LEP
}

\section{L3 Collaboration}

P. Achard ${ }^{\mathrm{t}}$, O. Adriani ${ }^{\mathrm{q}}$, M. Aguilar-Benitez ${ }^{\mathrm{y}}, \mathrm{J}$. Alcaraz $^{\mathrm{y}}$, G. Alemanni ${ }^{\mathrm{w}}$, J. Allaby ${ }^{\mathrm{r}}$, A. Aloisio $^{\text {ac }}$, M.G. Alviggi ac H. Anderhub ${ }^{\text {aw }}$, V.P. Andreev f,ah, F. Anselmo ${ }^{\text {h }}$, A. Arefiev ${ }^{\text {ab }}$, T. Azemoon ${ }^{\text {c }}$, T. Aziz ${ }^{\text {i }}$, P. Bagnaia ${ }^{a m}$, A. Bajo ${ }^{y}$, G. Baksay ${ }^{z}$, L. Baksay $^{z}$, S.V. Baldew ${ }^{\text {b }}$, S. Banerjee ${ }^{\text {i }, ~ S w . ~ B a n e r j e e ~}{ }^{d}$, A. Barczyk ${ }^{\text {aw }, \text { au }}$, R. Barillère ${ }^{r}$, P. Bartalini ${ }^{\text {w }}$, M. Basile ${ }^{\text {h }}$, N. Batalova ${ }^{\text {at }}$, R. Battiston ${ }^{\text {ag }}$, A. Bay ${ }^{\text {w }}$, F. Becattini ${ }^{\text {q }}$, U. Becker ${ }^{\mathrm{m}}$, F. Behner ${ }^{\mathrm{aw}}$, L. Bellucci ${ }^{\mathrm{q}}$, R. Berbeco ${ }^{\mathrm{c}}$, J. Berdugo ${ }^{\mathrm{y}}$, P. Berges ${ }^{\mathrm{m}}$, B. Bertucci ${ }^{\mathrm{ag}}$, B.L. Betev ${ }^{\text {aw }}$, M. Biasini ${ }^{\mathrm{ag}}$, M. Biglietti ${ }^{\text {ac }}$, A. Biland ${ }^{\text {aw }}$, J.J. Blaising ${ }^{\mathrm{d}}$, S.C. Blyth ${ }^{\text {ai }}$, G.J. Bobbink ${ }^{\text {b }}$, A. Böhm ${ }^{\text {a }}$, L. Boldizsar ${ }^{1}$, B. Borgia ${ }^{\text {am }}$, S. Bottai ${ }^{\text {q }}$, D. Bourilkov ${ }^{\text {aw }}$, M. Bourquin ${ }^{\mathrm{t}}$, S. Braccini ${ }^{\mathrm{t}}$, J.G. Branson ${ }^{\text {ao }}$, F. Brochu ${ }^{\mathrm{d}}$, J.D. Burger ${ }^{\mathrm{m}}$, W.J. Burger ${ }^{\mathrm{ag}}$, X.D. Cai ${ }^{\mathrm{m}}$, M. Capell ${ }^{\mathrm{m}}$, G. Cara Romeo ${ }^{\mathrm{h}}$, G. Carlino ${ }^{\mathrm{ac}}$, A. Cartacci ${ }^{\mathrm{q}}$, J. Casaus ${ }^{\mathrm{y}}$, F. Cavallari ${ }^{\text {am }}$, N. Cavallo ${ }^{\text {aj }}$, C. Cecchi ${ }^{\mathrm{ag}}$, M. Cerrada ${ }^{\mathrm{y}}$, M. Chamizo ${ }^{\mathrm{t}}$, Y.H. Chang ${ }^{\text {ar }}$, M. Chemarin ${ }^{\mathrm{x}}$, A. Chen ${ }^{\text {ar }}$, G. Chen ${ }^{\mathrm{g}}$, G.M. Chen ${ }^{\mathrm{g}}$, H.F. Chen ${ }^{\mathrm{v}}$, H.S. Chen ${ }^{\mathrm{g}}$, G. Chiefari ${ }^{\text {ac }}$, L. Cifarelli ${ }^{\text {an }}$, F. Cindolo ${ }^{\text {h }}$, I. Clare ${ }^{\mathrm{m}}$, R. Clare ${ }^{\text {al }}$, G. Coignet ${ }^{\mathrm{d}}$, N. Colino ${ }^{\mathrm{y}}$, S. Costantini ${ }^{\text {am }}$, B. de la Cruz $^{\mathrm{y}}$, S. Cucciarelli ${ }^{\mathrm{ag}}$, R. de Asmundis ${ }^{\text {ac }}$, P. Déglon ${ }^{\mathrm{t}}, \mathrm{J}$. Debreczeni ${ }^{1}$, A. Degré ${ }^{\mathrm{d}}$, K. Dehmelt ${ }^{\mathrm{z}}$, K. Deiters ${ }^{\mathrm{au}}$, D. della Volpe ${ }^{\text {ac }}$, E. Delmeire ${ }^{t}$, P. Denes ${ }^{\text {ak }}$, F. DeNotaristefani ${ }^{\text {am }}$, A. De Salvo ${ }^{\text {aw }}$, M. Diemoz ${ }^{\text {am }}$, M. Dierckxsens ${ }^{b}$, C. Dionisi ${ }^{\text {am }}$, M. Dittmar ${ }^{\text {aw }}$, A. Doria ${ }^{\text {ac }}$, M.T. Dova $^{\mathrm{j}, 5}$,

D. Duchesneau ${ }^{d}$, M. Duda ${ }^{a}$, B. Echenard ${ }^{t}$, A. Eline ${ }^{r}$, A. El Hage ${ }^{a}$, H. El Mamouni ${ }^{\mathrm{x}}$, A. Engler ${ }^{\text {ai }}$, F.J. Eppling ${ }^{\mathrm{m}}$, P. Extermann ${ }^{\mathrm{t}}$, M.A. Falagan ${ }^{\mathrm{y}}$, S. Falciano ${ }^{\text {am }}$, A. Favara ${ }^{\text {af }}$, J. Fay ${ }^{\mathrm{x}}$, O. Fedin ${ }^{\text {ah }}$, M. Felcini ${ }^{\text {aw }}$, T. Ferguson ${ }^{\text {ai }}$, H. Fesefeldt ${ }^{\mathrm{a}}$, E. Fiandrini ${ }^{\mathrm{ag}}$,

J.H. Field ${ }^{\mathrm{t}}$, F. Filthaut ${ }^{\mathrm{ae}}$, P.H. Fisher ${ }^{\mathrm{m}}$, W. Fisher ${ }^{\text {ak }}$, I. Fisk ${ }^{\mathrm{ao}}$, G. Forconi ${ }^{\mathrm{m}}$, K. Freudenreich ${ }^{\text {aw }}$, C. Furetta ${ }^{\text {aa }}$, Yu. Galaktionov ${ }^{\text {ab, }}{ }^{\text {, S.N. Ganguli }}{ }^{\mathrm{i}}$, P. Garcia-Abia ${ }^{\mathrm{y}}$, M. Gataullin ${ }^{\text {af }}$, S. Gentile ${ }^{\text {am }}$, S. Giagu ${ }^{\text {am }}$, Z.F. Gong ${ }^{\mathrm{v}}$, G. Grenier ${ }^{\mathrm{x}}$, O. Grimm ${ }^{\mathrm{aw}}$, M.W. Gruenewald ${ }^{\mathrm{p}}$, M. Guida ${ }^{\text {an }}$, V.K. Gupta ${ }^{\mathrm{ak}}$, A. Gurtu ${ }^{\mathrm{i}}$, L.J. Gutay ${ }^{\text {at }}$, D. Haas ${ }^{\mathrm{e}}$, D. Hatzifotiadou ${ }^{\text {h }}$, T. Hebbeker ${ }^{\text {a }}$, A. Hervé ${ }^{r}$, J. Hirschfelder ${ }^{\text {ai }}$, H. Hofer ${ }^{\text {aw }}$, M. Hohlmann ${ }^{\mathrm{z}}$, G. Holzner ${ }^{\text {aw }}$, S.R. Hou ${ }^{\text {ar }}$, B.N. Jin ${ }^{\mathrm{g}}$, P. Jindal ${ }^{\mathrm{n}}$, L.W. Jones ${ }^{\mathrm{c}}$, 
P. de Jong ${ }^{\text {b }}$, I. Josa-Mutuberría ${ }^{y}$, M. Kaur ${ }^{\text {n }}$, M.N. Kienzle-Focacci ${ }^{\text {t }}$ J.K. Kim ${ }^{\text {aq }}$, J. Kirkby ${ }^{\mathrm{r}}$, W. Kittel ${ }^{\text {ae }}$, A. Klimentov ${ }^{\mathrm{m}, \mathrm{ab}}$, A.C. König ${ }^{\text {ae }}$, M. Kopal ${ }^{\text {at }}$, V. Koutsenko ${ }^{\mathrm{m}, \mathrm{ab}}$, M. Kräber ${ }^{\text {aw }}$, R.W. Kraemer ${ }^{\text {ai }}$, A. Krüger ${ }^{\text {av }}$, A. Kunin ${ }^{\text {m }}$, P. Ladron de Guevara ${ }^{y}$, I. Laktineh ${ }^{\mathrm{x}}$, G. Landi ${ }^{\mathrm{q}}$, M. Lebeau ${ }^{\mathrm{r}}$, A. Lebedev ${ }^{\mathrm{m}}$, P. Lebrun ${ }^{\mathrm{x}}$, P. Lecomte ${ }^{\text {aw }}$, P. Lecoq ${ }^{r}$, P. Le Coultre ${ }^{\text {aw }}$, J.M. Le Goff ${ }^{\mathrm{r}}$, R. Leiste ${ }^{\text {av }}$, M. Levtchenko ${ }^{\text {aa }}$, P. Levtchenko ah ${ }^{\text {ah }}$ C. Li ${ }^{v}$, S. Likhoded ${ }^{\text {av }}$, C.H. Lin ${ }^{\text {ar }}$, W.T. Lin ${ }^{\text {ar }}$, F.L. Linde ${ }^{\text {b }}$, L. Lista ${ }^{\text {ac }}$, Z.A. Liu ${ }^{\mathrm{g}}$, W. Lohmann ${ }^{\text {av }}$, E. Longo ${ }^{\text {am }}$, Y.S. Lu ${ }^{\mathrm{g}}$, C. Luci ${ }^{\text {am }}$, L. Luminari ${ }^{\text {am }}$, W. Lustermann ${ }^{\mathrm{aw}}$, W.G. Ma ${ }^{\mathrm{v}}$, L. Malgeri ${ }^{\mathrm{r}}$, A. Malinin ${ }^{\mathrm{ab}}$, C. Maña ${ }^{\mathrm{y}}$, J. Mans $^{\mathrm{ak}}$, J.P. Martin x , F. Marzano am ${ }^{\text {a }}$ K. Mazumdar ${ }^{\text {i, R.R. McNeil }}{ }^{\text {f }}$, S. Mele ${ }^{\text {r,ac }}$, L. Merola ${ }^{\text {ac }}$, M. Meschini ${ }^{\mathrm{q}}$, W.J. Metzger ${ }^{\text {ae }}$, A. Mihul ${ }^{\mathrm{k}}$, H. Milcent ${ }^{\mathrm{r}}$, G. Mirabelli ${ }^{\text {am }}$, J. Mnich ${ }^{\mathrm{a}}$, G.B. Mohanty ${ }^{\text {i }}$, G.S. Muanza ${ }^{\mathrm{x}}$, A.J.M. Muijs ${ }^{\mathrm{b}}$, B. Musicar ${ }^{\mathrm{ac}}$, M. Musy ${ }^{\mathrm{am}}, \mathrm{S}$ S. Nagy ${ }^{\mathrm{o}}$, S. Natale ${ }^{\mathrm{t}}$, M. Napolitano ${ }^{\text {ac }}$, F. Nessi-Tedaldi ${ }^{\text {aw }}$, H. Newman ${ }^{\text {af }}$, A. Nisati ${ }^{\text {am }}$, T. Novak ${ }^{\text {ae }}$, H. Nowak ${ }^{\text {av }}$, R. Ofierzynski ${ }^{\text {aw }}$, G. Organtini ${ }^{\text {am }}$, I. Pal $^{\text {at }}$, C. Palomares ${ }^{y}$, P. Paolucci ${ }^{\text {ac }}$, R. Paramatti ${ }^{\text {am }}$, G. Passaleva ${ }^{\mathrm{q}}$, S. Patricelli ${ }^{\text {ac }}$, T. Paul $^{\mathrm{j}}$, M. Pauluzzi $^{\mathrm{ag}}$, C. Paus ${ }^{\mathrm{m}}$, F. Pauss ${ }^{\text {aw }}$, M. Pedace ${ }^{\mathrm{am}}$, S. Pensotti ${ }^{\text {aa }}$, D. Perret-Gallix ${ }^{\mathrm{d}}$, D. Piccolo ${ }^{\text {ac }}$, F. Pierella ${ }^{\text {h}}$, M. Pioppi ${ }^{\text {ag }}$, P.A. Piroué ${ }^{a k}$, E. Pistolesi ${ }^{\text {aa }}$, V. Plyaskin ${ }^{\text {ab }}$, M. Pohl ${ }^{t}$, V. Pojidaev ${ }^{\mathrm{q}}$, J. Pothier ${ }^{\mathrm{r}}$, D. Prokofiev ${ }^{\text {ah }}$, G. Rahal-Callot ${ }^{\text {aw }}$, M.A. Rahaman ${ }^{\mathrm{i}}$, P. Raics ${ }^{\text {o }}$, N. Raja ${ }^{\mathrm{i}}$, R. Ramelli ${ }^{\text {aw }}$, P.G. Rancoita ${ }^{\text {aa }}$, R. Ranieri ${ }^{\mathrm{q}}$, A. Raspereza ${ }^{\text {av }}$, P. Razis ${ }^{\text {ad }}$, D. Ren ${ }^{\text {aw }}$, M. Rescigno ${ }^{\text {am }}$, S. Reucroft ${ }^{\mathrm{j}}$, S. Riemann ${ }^{\text {av }}$, K. Riles ${ }^{\text {c }}$, B.P. Roe ${ }^{\mathrm{c}}$, L. Romero ${ }^{\mathrm{y}}$, A. Rosca ${ }^{\text {av }}$, C. Rosemann ${ }^{\mathrm{a}}$, C. Rosenbleck ${ }^{\mathrm{a}}$, S. Rosier-Lees ${ }^{\mathrm{d}}$, S. Roth ${ }^{\mathrm{a}}$, J.A. Rubio ${ }^{\text {r }}$, G. Ruggiero ${ }^{\text {q }}$, H. Rykaczewski ${ }^{\text {aw }}$, A. Sakharov ${ }^{\text {aw }}$, S. Saremi ${ }^{\text {f }}$, S. Sarkar ${ }^{\text {am }}$, J. Salicio $^{\text {r }}$, E. Sanchez ${ }^{y}$, C. Schäfer ${ }^{r}$, V. Schegelsky ${ }^{\text {ah }}$, H. Schopper ${ }^{\text {u }}$, D.J. Schotanus ${ }^{\text {ae }}$, C. Sciacca ${ }^{\text {ac }}$, L. Servoli ${ }^{\text {ag }}$, S. Shevchenko ${ }^{\text {af }}$, N. Shivarov ${ }^{\text {ap }}$, V. Shoutko ${ }^{\mathrm{m}}$, E. Shumilov ${ }^{\text {ab }}$, A. Shvorob af, D. Son ${ }^{\text {aq }}$, C. Souga ${ }^{x}$, P. Spillantini ${ }^{q}$, M. Steuer ${ }^{\mathrm{m}}$, D.P. Stickland ${ }^{\text {ak }}$, B. Stoyanov ap, A. Straessner ${ }^{\mathrm{t}}$, K. Sudhakar ${ }^{\mathrm{i}}$, G. Sultanov ${ }^{\text {ap }}$, L.Z. Sun ${ }^{\mathrm{v}}$, S. Sushkov ${ }^{\mathrm{a}}$, H. Suter ${ }^{\text {aw }}$, J.D. Swain ${ }^{\mathrm{j}}$, Z. Szillasi ${ }^{\mathrm{z}, 3}$, X.W. Tang ${ }^{\mathrm{g}}$, P. Tarjan ${ }^{\mathrm{o}}$, L. Tauscher ${ }^{\mathrm{e}}$, L. Taylor ${ }^{\mathrm{j}}$, B. Tellili ${ }^{\mathrm{x}}, \mathrm{D}$. Teyssier ${ }^{\mathrm{x}}$, C. Timmermans ${ }^{\text {ae }}$, Samuel C.C. Ting ${ }^{\mathrm{m}}$, S.M. Ting ${ }^{\mathrm{m}}$, S.C. Tonwar ${ }^{\mathrm{i}}$, J. Tóth ${ }^{1}$, C. Tully ${ }^{\mathrm{ak}}$, K.L. Tung ${ }^{\mathrm{g}}$, J. Ulbricht ${ }^{\text {aw }}$, E. Valente ${ }^{\text {am }}$, R.T. Van de Walle ${ }^{\text {ae }}$, R. Vasquez ${ }^{\text {at }}$, V. Veszpremi ${ }^{\mathrm{z}}$, G. Vesztergombi ${ }^{1}$, I. Vetlitsky ${ }^{\text {ab }}$, G. Viertel ${ }^{\text {aw }}$, S. Villa ${ }^{\text {al }}$, M. Vivargent ${ }^{\text {d }}$, S. Vlachos $^{\mathrm{e}}$, I. Vodopianov $^{z}$, H. Vogel ${ }^{\text {ai }}, H$. Vogt ${ }^{\text {av }}$, I. Vorobiev ${ }^{\text {ai,ab }}$, A.A. Vorobyov ${ }^{\text {ah }}$, M. Wadhwa ${ }^{\mathrm{e}}$, Q. Wang ${ }^{\text {ae }}$, X.L. Wang ${ }^{\mathrm{v}}$, Z.M. Wang ${ }^{\mathrm{v}}$, M. Weber ${ }^{\mathrm{r}}$, S. Wynhoff ${ }^{\mathrm{ak}}$, L. Xia ${ }^{\text {af }}$, Z.Z. Xu ${ }^{\mathrm{v}}$,

J. Yamamoto $^{\text {c }}$, B.Z. Yang ${ }^{v}$, C.G. Yang ${ }^{\mathrm{g}}$, H.J. Yang ${ }^{\mathrm{c}}$, M. Yang ${ }^{\mathrm{g}}$, S.C. Yeh ${ }^{\text {as }}$, An. Zalite ${ }^{\text {ah }}$, Yu. Zalite ${ }^{\text {ah }}$, Z.P. Zhang ${ }^{v}$, J. Zhao ${ }^{v}$, G.Y. Zhu ${ }^{\mathrm{g}}$, R.Y. Zhu ${ }^{\text {af }}$, H.L. Zhuang ${ }^{\mathrm{g}}$, A. Zichichi ${ }^{\mathrm{h}, \mathrm{r}, \mathrm{s}}$, B. Zimmermann ${ }^{\text {aw }}$, M. Zöller ${ }^{\mathrm{a}}$

\footnotetext{
a III. Physikalisches Institut, RWTH, D-52056 Aachen, Germany ${ }^{1}$

${ }^{\mathrm{b}}$ National Institute for High Energy Physics, NIKHEF, and University of Amsterdam, NL-1009 DB Amsterdam, The Netherlands c University of Michigan, Ann Arbor, MI 48109, USA

d Laboratoire d'Annecy-le-Vieux de Physique des Particules, LAPP, IN2P3-CNRS, BP 110, F-74941 Annecy-le-Vieux cedex, France
} 
e Institute of Physics, University of Basel, CH-4056 Basel, Switzerland

${ }^{\mathrm{f}}$ Louisiana State University, Baton Rouge, LA 70803, USA

g Institute of High Energy Physics, IHEP, 100039 Beijing, China 6

${ }^{\mathrm{h}}$ University of Bologna and INFN, Sezione di Bologna, I-40126 Bologna, Italy

${ }^{\mathrm{i}}$ Tata Institute of Fundamental Research, Mumbai (Bombay) 400 005, India

$\mathrm{j}$ Northeastern University, Boston, MA 02115, USA

${ }^{\mathrm{k}}$ Institute of Atomic Physics and University of Bucharest, R-76900 Bucharest, Romania

${ }^{1}$ Central Research Institute for Physics of the Hungarian Academy of Sciences, H-1525 Budapest 114, Hungary ${ }^{2}$

$\mathrm{m}$ Massachusetts Institute of Technology, Cambridge, MA 02139, USA

${ }^{\mathrm{n}}$ Panjab University, Chandigarh 160 014, India

${ }^{\circ}$ KLTE-ATOMKI, H-4010 Debrecen, Hungary ${ }^{3}$

$\mathrm{p}$ Department of Experimental Physics, University College Dublin, Belfield, Dublin 4, Ireland

q INFN, Sezione di Firenze and University of Florence, I-50125 Florence, Italy

${ }^{\mathrm{r}}$ European Laboratory for Particle Physics, CERN, CH-1211 Geneva 23, Switzerland

s World Laboratory, FBLJA Project, CH-1211 Geneva 23, Switzerland

${ }^{\mathrm{t}}$ University of Geneva, CH-1211 Geneva 4, Switzerland

u University of Hamburg, D-22761 Hamburg, Germany

${ }^{v}$ Chinese University of Science and Technology, USTC, Hefei, Anhui 230 029, China ${ }^{6}$

${ }^{\mathrm{w}}$ University of Lausanne, CH-1015 Lausanne, Switzerland

${ }^{\mathrm{x}}$ Institut de Physique Nucléaire de Lyon, IN2P3-CNRS, Université Claude Bernard, F-69622 Villeurbanne, France

y Centro de Investigaciones Energéticas, Medioambientales y Tecnológicas, CIEMAT, E-28040 Madrid, Spain ${ }^{4}$

${ }^{\mathrm{z}}$ Florida Institute of Technology, Melbourne, FL 32901, USA

aa INFN, Sezione di Milano, I-20133 Milan, Italy

ab Institute of Theoretical and Experimental Physics, ITEP, Moscow, Russia

${ }^{\text {ac }}$ INFN, Sezione di Napoli and University of Naples, I-80125 Naples, Italy

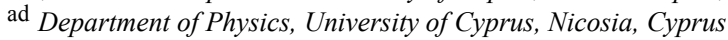

ae Radboud University and NIKHEF, NL-6525 ED Nijmegen, The Netherlands

af California Institute of Technology, Pasadena, CA 91125, USA

ag INFN, Sezione di Perugia and Università Degli Studi di Perugia, I-06100 Perugia, Italy

ah Nuclear Physics Institute, St. Petersburg, Russia

ai Carnegie Mellon University, Pittsburgh, PA 15213, USA

aj INFN, Sezione di Napoli and University of Potenza, I-85100 Potenza, Italy

${ }^{\mathrm{ak}}$ Princeton University, Princeton, NJ 08544, USA

al University of Californa, Riverside, CA 92521, USA

am INFN, Sezione di Roma and University of Rome, "La Sapienza", I-00185 Rome, Italy

an University and INFN, Salerno, I-84100 Salerno, Italy

ao University of California, San Diego, CA 92093, USA

ap Bulgarian Academy of Sciences, Central Lab. of Mechatronics and Instrumentation, BU-1113 Sofia, Bulgaria

aq The Center for High Energy Physics, Kyungpook National University, 702-701 Taegu, Republic of Korea

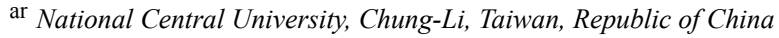

as Department of Physics, National Tsing Hua University, Taiwan, Republic of China

at Purdue University, West Lafayette, IN 47907, USA

au Paul Scherrer Institut, PSI, CH-5232 Villigen, Switzerland av DESY, D-15738 Zeuthen, Germany

${ }^{\text {aw }}$ Eidgenössische Technische Hochschule, ETH Zürich, CH-8093 Zürich, Switzerland

Received 1 March 2005; received in revised form 13 May 2005; accepted 16 May 2005

Available online 8 June 2005

Editor: L. Rolandi

\section{Abstract}

The cross section for open-beauty production in photon-photon collisions is measured using the whole high-energy and high-luminosity data sample collected by the L3 detector at LEP. This corresponds to $627 \mathrm{pb}^{-1}$ of integrated luminosity for 
electron-positron centre-of-mass energies from 189 to $209 \mathrm{GeV}$. Events containing b quarks are identified through their semileptonic decay into electrons or muons. The $\mathrm{e}^{+} \mathrm{e}^{-} \rightarrow \mathrm{e}^{+} \mathrm{e}^{-} \mathrm{b} \bar{b}$ cross section is measured within our fiducial volume and then extrapolated to the full phase space. These results are found to be in significant excess with respect to Monte Carlo predictions and next-to-leading order QCD calculations.

(c) 2005 Published by Elsevier B.V.

\section{Introduction}

The production of $\mathrm{b}$ quarks through hard processes constitutes a unique environment for the study of perturbative QCD, as the mass of the b quark, $m_{\mathrm{b}}$, largely exceeds the typical non-perturbative scale of hadronic interactions. High-energy hadron colliders are copious sources of $b$ quarks and therefore extensive experimental studies and QCD calculations have been performed. Much debate has taken place on the apparent disagreement between the measured cross section for b-quark production in $p \bar{p}$ collisions at the Tevatron [1] and the next-to-leading order (NLO) QCD calculations [2]. The first measurements of open beauty production in $\mathrm{e}^{ \pm} \mathrm{p}$ collisions at HERA were found to be markedly higher than NLO QCD predictions [3]. Some more recent measurements were in better agreement [4], while others still showed an excess $[5,6]$. A comparison of these different measurements with NLO QCD predictions is shown in Ref. [6].

Photon-photon collisions at $\mathrm{e}^{+} \mathrm{e}^{-}$colliders also give access to the hard production of $\mathrm{b}$ quarks. The LEP $\mathrm{e}^{+} \mathrm{e}^{-}$centre-of-mass energy, $\sqrt{s}$, was around $200 \mathrm{GeV}$. In this environment $\mathrm{b}$ quarks are expected to be produced with comparable rates by the direct and single-resolved processes [7], illustrated in Fig. 1. The main contribution to the resolved-photon cross section is the photon-gluon fusion process. The rates of both

\footnotetext{
${ }^{1}$ Supported by the German Bundesministerium für Bildung, Wissenschaft, Forschung und Technologie.

2 Supported by the Hungarian OTKA fund under contract No. T019181, F023259 and T037350.

3 Also supported by the Hungarian OTKA fund under contract No. T026178.

${ }^{4}$ Supported also by the Comisión Interministerial de Ciencia y Tecnología.

5 Also supported by CONICET and Universidad Nacional de La Plata, CC 67, 1900 La Plata, Argentina.

6 Supported by the National Natural Science Foundation of China.
}
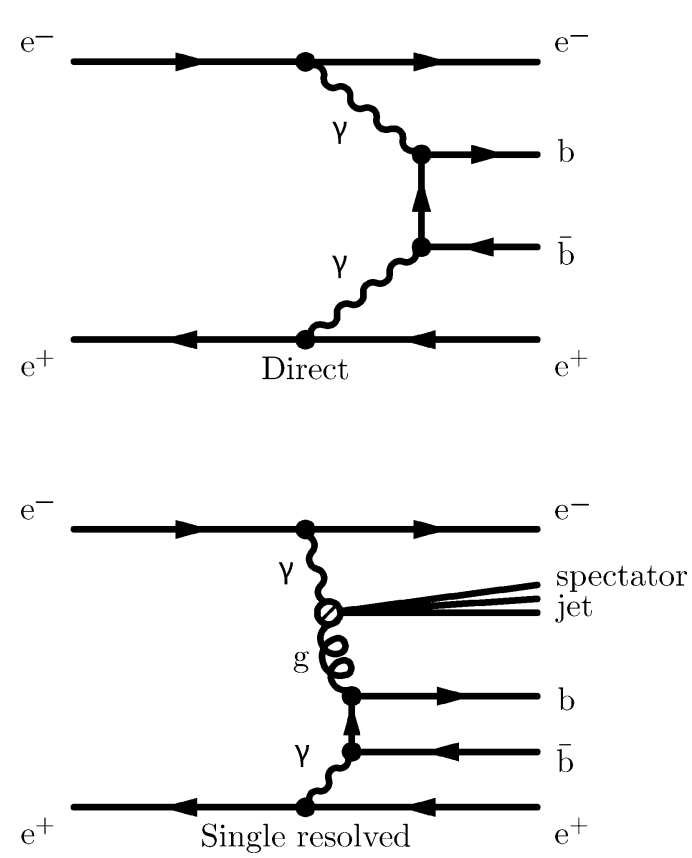

Fig. 1. Dominant diagrams contributing to open-beauty production in photon-photon collisions at LEP.

the direct and the single-resolved process depend on $m_{\mathrm{b}}$, while the latter also depends on the gluon density in the photon.

The first measurement of the cross section for the $\mathrm{e}^{+} \mathrm{e}^{-} \rightarrow \mathrm{e}^{+} \mathrm{e}^{-} \mathrm{b} \overline{\mathrm{b}} \mathrm{X}$ process was published by the $\mathrm{L} 3$ Collaboration using $410 \mathrm{pb}^{-1}$ of data collected at $\sqrt{s}=189-202 \mathrm{GeV}$ [8]. The results were found to be in excess of the QCD prediction by a factor of three. Since these first findings, compatible preliminary results were obtained by other LEP collaborations [9]. In this Letter, we extend our measurement to the whole high-energy and high-luminosity data sample collected at LEP with the L3 detector [10], corresponding to $627 \mathrm{pb}^{-1}$ at $\sqrt{s}=189-209 \mathrm{GeV}$.

Hadronic events from photon-photon interactions are selected through their specific multiplicity and topology. The production of $\mathrm{b}$ quarks is then tagged by 
the detection of electrons ${ }^{7}$ or muons from their semileptonic decays. The cross section of the $\mathrm{e}^{+} \mathrm{e}^{-} \rightarrow$ $\mathrm{e}^{+} \mathrm{e}^{-} \mathrm{b} \overline{\mathrm{b}} \mathrm{X}$ process is measured in a phase space which reflects the energy thresholds used in the analyses and the fiducial volume for lepton identification: the lepton momentum must exceed $2 \mathrm{GeV}$ and the angle, $\theta$, between the leptons and the beam line must satisfy $|\cos \theta|<0.725$ for electrons and $|\cos \theta|<0.8$ for muons, respectively. For the first time the experimental results are compared to Monte Carlo predictions in this fiducial volume. An extrapolation factor is then applied to compare the measured cross section with the QCD predictions in the full phase space.

\section{Monte Carlo simulations}

The PYTHIA [11] Monte Carlo generator is used to model hadron production in photon-photon collisions. Final states without b quarks are generated with massless matrix elements [12] while massive matrix elements are used for b-quark production. Resolved processes are described by means of the SaS1d parton density function [13]. The photon-photon luminosity function is implemented in the equivalent photon approximation [14] with a cutoff for the virtuality of the interacting photons $Q^{2}<m_{\rho}^{2}$.

Potential backgrounds are simulated by the following Monte Carlo generators: JAMVG [15] for the $\mathrm{e}^{+} \mathrm{e}^{-} \rightarrow \mathrm{e}^{+} \mathrm{e}^{-} \tau^{+} \tau^{-}$process, PYTHIA for $\mathrm{e}^{+} \mathrm{e}^{-} \rightarrow$ $\mathrm{q} \overline{\mathrm{q}}$, KORALZ [16] for $\mathrm{e}^{+} \mathrm{e}^{-} \rightarrow \tau^{+} \tau^{-}$and KORALW [17] for $\mathrm{e}^{+} \mathrm{e}^{-} \rightarrow \mathrm{W}^{+} \mathrm{W}^{-}$.

The L3 detector is simulated using the GEANT [18] and GHEISHA [19] packages. Monte Carlo events are then reconstructed in the same way as the data. Timedependent detector inefficiencies, as monitored during the data-taking period, are included in the simulations.

\section{Event selection}

The selection of events originating from the $\mathrm{e}^{+} \mathrm{e}^{-} \rightarrow \mathrm{e}^{+} \mathrm{e}^{-} \mathrm{b} \overline{\mathrm{b} X}$ process is unchanged with respect to Ref. [8]. Hadrons produced in photon-photon col-

\footnotetext{
7 Throughout this Letter, the term 'electron' stands for both electrons and positrons.
}

lisions are selected by means of three criteria. First, at least five charged tracks are required, thus suppressing background from the $\mathrm{e}^{+} \mathrm{e}^{-} \rightarrow \mathrm{e}^{+} \mathrm{e}^{-} \tau^{+} \tau^{-}$and $\mathrm{e}^{+} \mathrm{e}^{-} \rightarrow \tau^{+} \tau^{-}$processes. Second, the visible energy of the event, $E_{\mathrm{vis}}$, is required to satisfy $E_{\mathrm{vis}}<\sqrt{s} / 3$, in order to reject events from the $\mathrm{e}^{+} \mathrm{e}^{-} \rightarrow \mathrm{q} \overline{\mathrm{q}}$ annihilation process and further eliminate events from the $\mathrm{e}^{+} \mathrm{e}^{-} \rightarrow \tau^{+} \tau^{-}$process. Finally, possible instrumental background and uncertainties in the trigger procedure are reduced by requiring the event visible mass, $W_{\text {vis }}$, to satisfy $W_{\text {vis }}>3 \mathrm{GeV}$. $W_{\text {vis }}$ is calculated from the four momenta of reconstructed tracks and of isolated calorimetric clusters. In this calculation, the pion mass is associated to the tracks while the clusters are treated as massless. Clusters in the low-angle luminosity monitor are included in this calculation.

In addition to these cuts, the analysis is restricted to events with small photon virtuality by removing events with clusters with energy greater than $0.2 \sqrt{s}$ in the low-angle calorimeter, covering a polar angle from $1.4^{\circ}$ to $3.7^{\circ}$. This criteria corresponds to retaining quasi-real photons with $\left\langle Q^{2}\right\rangle \simeq 0.015 \mathrm{GeV}^{2}$.

About two million photon-photon events are selected by these cuts, with a background contamination of $0.1 \%$. Events are further analysed if they have an identified electron or muon.

Electrons are identified as clusters in the electromagnetic calorimeter in the polar angular range $|\cos \theta|<0.725$ with energy above $2 \mathrm{GeV}$. They should match a track and have a shower profile compatible with that expected for an electromagnetic shower. The ratio $E_{\mathrm{t}} / p_{\mathrm{t}}$ between the projection of the cluster energy on the plane transverse to the beams and the transverse momentum of the track is required to be compatible with unity. Electrons due to photon conversion are suppressed by requiring the distance of closest approach, in the transverse plane, of the track to the mean $\mathrm{e}^{+} \mathrm{e}^{-}$collision point in the transverse plane to be less than $0.5 \mathrm{~mm}$ and the invariant mass of the electron candidate and of the closest track, considered as an electron, to be greater than $0.1 \mathrm{GeV}$.

These cuts select 82 events with electron candidates in the $217 \mathrm{pb}^{-1}$ of data collected at $\sqrt{s}=$ 202-209 GeV, which together with the 137 events previously selected in the data at $\sqrt{s}=189-202 \mathrm{GeV}$ [8] give a total of 219 events with an expected background of $2.0 \%$ from the $\mathrm{e}^{+} \mathrm{e}^{-} \rightarrow \mathrm{q} \overline{\mathrm{q}}$ and $\mathrm{e}^{+} \mathrm{e}^{-} \rightarrow \tau^{+} \tau^{-}$ processes and a signal efficiency of $1.3 \%$. 
Muon candidates are selected from tracks in the muon spectrometer in the range $|\cos \theta|<0.8$. A minimal muon momentum of $2 \mathrm{GeV}$ is required to ensure the muons reach the spectrometer after having crossed the calorimeters. The background from annihilation processes is suppressed by requiring the muon momentum to be less than $0.1 \sqrt{s}$. Background from cosmic muons is rejected by requiring the muons to be associated with a signal in the scintillator time-offlight system in time with the beam crossing.

After these cuts, 166 events with muon candidates are selected in data with $\sqrt{s}=202-209 \mathrm{GeV}$. Including the 269 events previously selected at $\sqrt{s}=$ 189-202 GeV [8], a total of 435 events with muons are retained. The estimated background from the $\mathrm{e}^{+} \mathrm{e}^{-} \rightarrow$ $\mathrm{q} \overline{\mathrm{q}}, \mathrm{e}^{+} \mathrm{e}^{-} \rightarrow \tau^{+} \tau^{-}$and $\mathrm{e}^{+} \mathrm{e}^{-} \rightarrow \mathrm{e}^{+} \mathrm{e}^{-} \tau^{+} \tau^{-}$is $5.7 \%$ and the signal efficiency is $2.2 \%$.

Fig. 2 presents the $W_{\text {vis }}$ spectra of the selected events for the electron and muon samples.

\section{Results}

The cross section for the $\mathrm{e}^{+} \mathrm{e}^{-} \rightarrow \mathrm{e}^{+} \mathrm{e}^{-} \mathrm{b} \overline{\mathrm{b}} \mathrm{X}$ process is determined from the distribution of the transverse momentum of the lepton with respect to the nearest jet, $P_{\mathrm{t}}$. As a consequence of the large value of $m_{\mathrm{b}}$, the distribution of this variable is enhanced for high values as compared to the background. The jets are reconstructed using the JADE algorithm [20] with $y_{\text {cut }}=0.1$. The identified lepton is not included in the jet. Fig. 3 presents the observed distributions of $P_{\mathrm{t}}$ for electrons and muons.

The data distributions are fitted using the leastsquares method to the sum of four contributions, whose shapes are fixed by Monte Carlo simulations. The first describes the background from annihilation processes and the $\mathrm{e}^{+} \mathrm{e}^{-} \rightarrow \mathrm{e}^{+} \mathrm{e}^{-} \tau^{+} \tau^{-}$reaction. Its normalisation, $N_{\mathrm{bkg}}$, is fixed to the Monte Carlo predictions listed in Table 1. The three other contributions are those from b quarks, c quarks and lighter flavours. Their normalisations, $N_{\mathrm{b} \overline{\mathrm{b}}}, N_{\mathrm{c} \overline{\mathrm{c}}}$ and $N_{\mathrm{uds}}$, respectively, are the free parameters of the fit. The results of the fits are given in Table 1: a b-quark fraction of $46.2 \pm 5.1 \%$ is observed for electrons and $41.2 \pm 3.8 \%$ for muons, where the uncertainties are statistical. The $\chi^{2}$ per degree of freedom of the fits is acceptable, with values of $13.7 / 6$ for electrons and $6.4 / 6$ for muons. A corre-
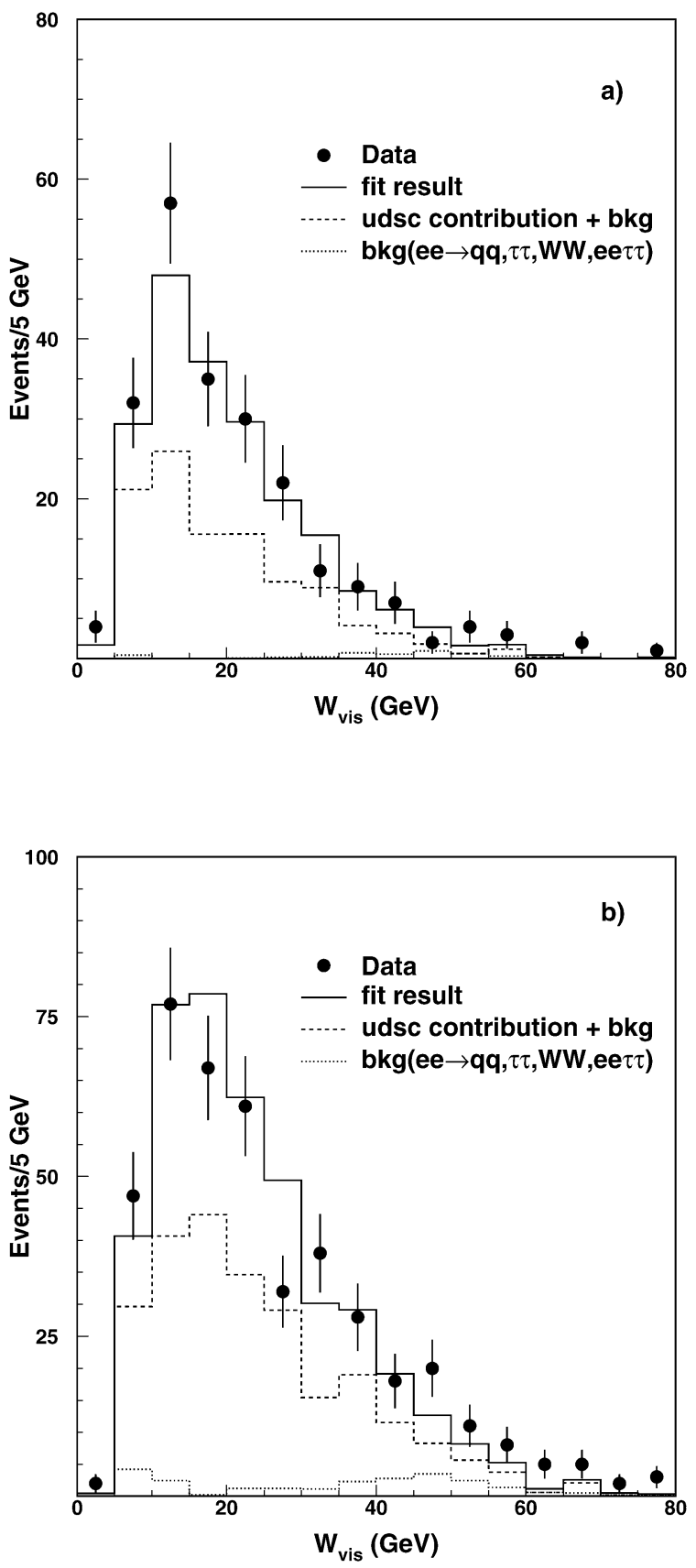

Fig. 2. Visible-mass spectra for the selected $\mathrm{e}^{+} \mathrm{e}^{-} \rightarrow \mathrm{e}^{+} \mathrm{e}^{-}$hadrons events containing (a) an electron or (b) a muon candidate at $\sqrt{s}=189-209 \mathrm{GeV}$. The points are the data while the dotted line represents the background from the $\mathrm{e}^{+} \mathrm{e}^{-} \rightarrow \mathrm{q} \overline{\mathrm{q}}, \mathrm{e}^{+} \mathrm{e}^{-} \rightarrow \tau^{+} \tau^{-}$, $\mathrm{e}^{+} \mathrm{e}^{-} \rightarrow \mathrm{W}^{+} \mathrm{W}^{-}$and $\mathrm{e}^{+} \mathrm{e}^{-} \rightarrow \mathrm{e}^{+} \mathrm{e}^{-} \tau^{+} \tau^{-}$processes. The dashed lines are the sum of this background and the light-quark contribution, while the solid lines also include b-quark production. The normalisation follows from the fit discussed in the text. 

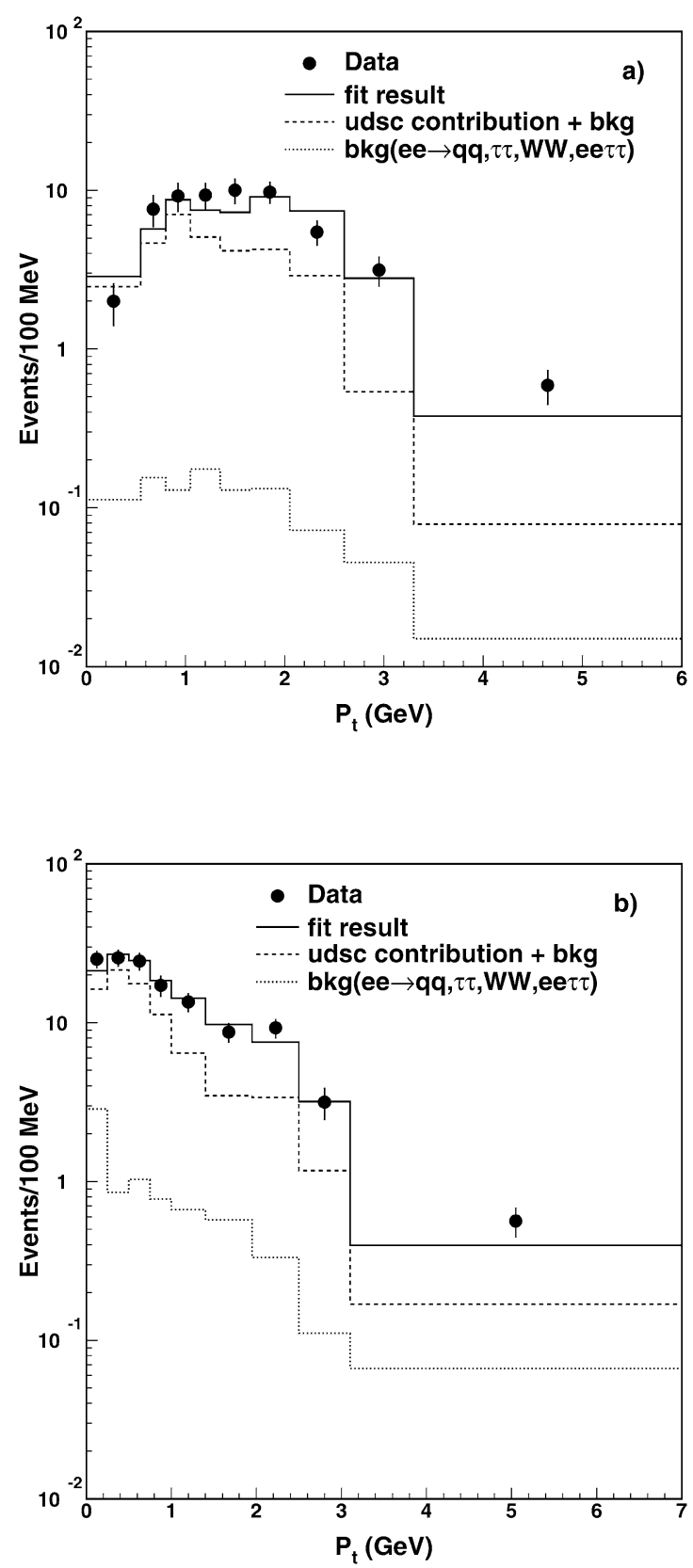

Fig. 3. Distributions of the transverse momentum of (a) the electron candidate and (b) the muon candidate with respect to the closest jet for the data and the results of the fit. The points are the data while the dotted line represents the background from the $\mathrm{e}^{+} \mathrm{e}^{-} \rightarrow \mathrm{q} \overline{\mathrm{q}}$, $\mathrm{e}^{+} \mathrm{e}^{-} \rightarrow \tau^{+} \tau^{-}, \mathrm{e}^{+} \mathrm{e}^{-} \rightarrow \mathrm{W}^{+} \mathrm{W}^{-}$and $\mathrm{e}^{+} \mathrm{e}^{-} \rightarrow \mathrm{e}^{+} \mathrm{e}^{-} \tau^{+} \tau^{-}$ processes. The dashed lines are the sum of this background and the light-quark contribution, while the solid lines also include b-quark production. The normalisation follows from the fit discussed in the text.
Table 1

Results of the fit to the distribution of the transverse momentum of the lepton with respect to the nearest jet. The fit parameters are constrained to be positive. The correlation between $N_{\mathrm{b} \overline{\mathrm{b}}}$ and $N_{\mathrm{c} \overline{\mathrm{c}}}$ is $75 \%$

\begin{tabular}{lcc}
\hline & Electrons & Muons \\
\hline$N_{\mathrm{bkg}}$ & 4.4 (fixed) & 24.8 (fixed) \\
$N_{\mathrm{b} \overline{\mathrm{b}}}$ & $94.3 \pm 18.3$ & $172.0 \pm 31.0$ \\
$N_{\mathrm{c} \overline{\mathrm{c}}}$ & $105.4 \pm 17.9$ & $220.5 \pm 35.4$ \\
$N_{\text {uds }}$ & $0.0_{-0.0}^{+12.0}$ & $0.0_{-0.0}^{+52.3}$ \\
$\chi^{2} /$ d.o.f. & $13.7 / 6$ & $6.4 / 6$ \\
\hline
\end{tabular}

lation coefficient of about $75 \%$ between $N_{\mathrm{b} \overline{\mathrm{b}}}$ and $N_{\mathrm{c} \overline{\mathrm{c}}}$ is observed. The results of the fits are also graphically shown in Fig. 3. Fig. 4 presents the distributions of the lepton momentum, transverse momentum and cosine of polar angle.

The measured fractions of $b$ quarks correspond to observed cross sections for the luminosity-averaged centre-of-mass energy $\langle\sqrt{s}\rangle=198 \mathrm{GeV}$ of

$$
\begin{gathered}
\sigma\left(\mathrm{e}^{+} \mathrm{e}^{-} \rightarrow \mathrm{e}^{+} \mathrm{e}^{-} \mathrm{b} \overline{\mathrm{b} X}\right)_{\text {electrons }}^{\text {observed }} \\
\quad=0.41 \pm 0.08 \pm 0.08 \mathrm{pb} \\
\sigma\left(\mathrm{e}^{+} \mathrm{e}^{-} \rightarrow \mathrm{e}^{+} \mathrm{e}^{-} \mathrm{b} \overline{\mathrm{b} X}\right)_{\text {muons }}^{\text {observed }} \\
\quad=0.56 \pm 0.10 \pm 0.10 \mathrm{pb} .
\end{gathered}
$$

The first uncertainties are statistical and the second systematic, and arise from the sources discussed below. These cross sections correspond to the phase space of the selected leptons, without any extrapolation: lepton momenta above $2 \mathrm{GeV}$ and polar angles in the ranges $|\cos \theta|<0.725$ for electrons and $|\cos \theta|<0.8$ for muons, respectively.

\section{Systematic uncertainties}

Several potential sources of systematic uncertainty are considered and their impact on the observed cross section is detailed in Table 2. The largest sources of uncertainty arises from the event-selection procedure and the Monte Carlo modelling of the detector response. Several components contribute to these uncertainties: the event-selection criteria, the lepton identification and the detector response and resolution on the energy and angular variables which identify the fiducial volume. The effect of these systematic uncertainties is estimated by varying the corresponding 

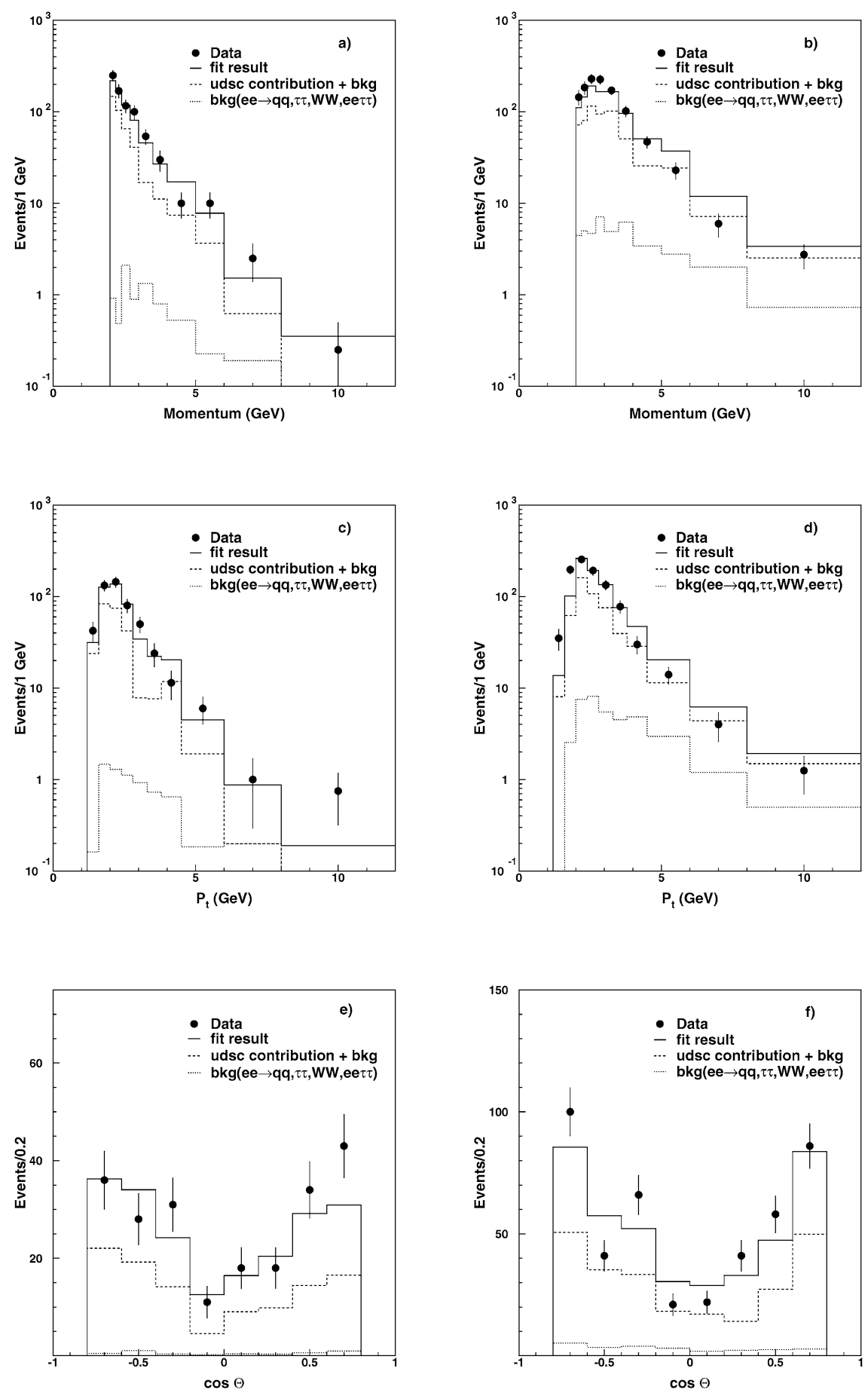

Fig. 4. Distribution of (a) the lepton momentum, (c) its transverse momentum and (e) the cosine of its polar angle for events containing electrons and (b), (d) and (f) for events containing muons. The points are the data while the dotted line represents the background from the $\mathrm{e}^{+} \mathrm{e}^{-} \rightarrow \mathrm{q} \overline{\mathrm{q}}$, $\mathrm{e}^{+} \mathrm{e}^{-} \rightarrow \tau^{+} \tau^{-}, \mathrm{e}^{+} \mathrm{e}^{-} \rightarrow \mathrm{W}^{+} \mathrm{W}^{-}$and $\mathrm{e}^{+} \mathrm{e}^{-} \rightarrow \mathrm{e}^{+} \mathrm{e}^{-} \tau^{+} \tau^{-}$processes. The dashed lines are the sum of this background and the light-quark contribution, while the solid lines also include b-quark production. The normalisation follows from the fit discussed in the text. 
Table 2

Systematic uncertainties on the observed values of the cross section of the process $\mathrm{e}^{+} \mathrm{e}^{-} \rightarrow \mathrm{e}^{+} \mathrm{e}^{-} \mathrm{b} \overline{\mathrm{b} X}$ for events tagged by electrons or muons. An additional uncertainty of $3 \%$ affects the extrapolation to the total cross section

\begin{tabular}{lcc}
\hline Source of uncertainty & \multicolumn{2}{c}{ Uncertainty on cross section (\%) } \\
\cline { 2 - 3 } & Electrons & Muons \\
\hline Event selection & 6.0 & 10.4 \\
Lepton identification & 7.9 & 2.2 \\
Fiducial volume & 12.3 & 10.0 \\
Jet reconstruction & 8.2 & 8.2 \\
Massive/massless charm & 3.0 & 3.0 \\
Trigger efficiency & 2.0 & 2.0 \\
Monte Carlo statistics & 1.6 & 1.4 \\
Direct/resolved ratio & 0.1 & 1.0 \\
Total & 18.3 & 17.2 \\
\hline
\end{tabular}

cuts and repeating the fits for the newly selected event samples. The second most important source of systematic uncertainty is the jet-reconstruction method. It is assessed by varying the value of $y_{\text {cut }}$ used in the reconstruction of the jets, and performing the fits for the different $P_{\mathrm{t}}$ distributions which are obtained after the corresponding variation of the jet direction. This variation also addresses uncertainties in the hadronisation process by excluding or adding soft clusters to the jets. The impact of the modelling of $\mathrm{c}$ quarks in the event generation is estimated by repeating fits by using Monte Carlo events generated with massive matrix elements. The trigger efficiency is determined from the data themselves and found to be $(95.6 \pm 2.0) \%$, this uncertainty is also propagated to the final results. The limited Monte Carlo statistics has a small impact on the total systematic uncertainty. In the fits, the signal events are produced in two separate samples for the direct and resolved processes and then combined in a $1: 1$ ratio [7]. Systematic uncertainties on this prediction are estimated by repeating the fits with ratios of $1: 2$ and $2: 1$.

\section{Discussion and conclusions}

The b-production cross sections measured in the phase space of the selected leptons are compared with the predictions obtained with the CASCADE Monte Carlo program [21]. This generator employs a backward-evolving parton cascade based on the
CCFM [22] equation. The most important difference as compared to NLO QCD calculations is the use of an unintegrated gluon density function taking explicitly into account the transverse momentum distribution of initial state gluons in hard scattering processes. In NLO QCD, all initial state partons have vanishing transverse momentum. CASCADE was shown [23] to give a consistent description of $b$-quark production at the Tevatron, whereas $\mathrm{H} 1$ electro-production data was found to be in excess by a factor of 2.6. Better agreement was found with ZEUS electro-production data.

The comparison of measurements and expectations in the actual phase space of the selected leptons has the advantage of providing an assessment of the agreement before any extrapolation is performed. Summing statistical and systematic uncertainties in quadrature, one finds

$$
\begin{aligned}
& \sigma\left(\mathrm{e}^{+} \mathrm{e}^{-} \rightarrow \mathrm{e}^{+} \mathrm{e}^{-} \mathrm{b} \overline{\mathrm{b} X}\right)_{\text {electrons }}^{\text {observed }}=0.41 \pm 0.11 \mathrm{pb}, \\
& \sigma\left(\mathrm{e}^{+} \mathrm{e}^{-} \rightarrow \mathrm{e}^{+} \mathrm{e}^{-} \mathrm{b} \overline{\mathrm{b} X}\right)_{\text {electrons }}^{\text {CASCADE }}=0.11 \pm 0.02 \mathrm{pb}, \\
& \sigma\left(\mathrm{e}^{+} \mathrm{e}^{-} \rightarrow \mathrm{e}^{+} \mathrm{e}^{-} \mathrm{b} \bar{b} \mathrm{X}\right)_{\text {muons }}^{\text {observed }}=0.56 \pm 0.14 \mathrm{pb}, \\
& \sigma\left(\mathrm{e}^{+} \mathrm{e}^{-} \rightarrow \mathrm{e}^{+} \mathrm{e}^{-} \mathrm{b} \overline{\mathrm{b} X}\right)_{\text {muons }}^{\text {CASCADE }}=0.14 \pm 0.02 \mathrm{pb},
\end{aligned}
$$

where the uncertainty on the CASCADE predictions corresponds to a variation of $m_{\mathrm{b}}$ in the range $4.75 \pm$ $0.25 \mathrm{GeV}$ [24]. A disagreement of about three standard deviations is observed for both flavours of the final-state leptons. This disagreement is mostly due to the overall normalisation of the sample rather than to a difference in shape of the most relevant kinematic variables, as also shown in Figs. 3 and 4.

The total cross section for open-beauty production in photon-photon collisions is determined by an extrapolation of the observed cross section to the full phase space of the process and by correcting for the semi-leptonic branching ratio of $b$ quarks. The extrapolation factors are determined with the PYTHIA Monte Carlo program, and similar results are obtained if the CASCADE Monte Carlo is used. Their difference, which amounts to $3 \%$, is considered as an additional systematic uncertainty. The experimental uncertainties on the semi-leptonic branching ratio of $b$ quarks [25] is also propagated to the measurement. 
The results for the electron and muon final states read

$$
\begin{aligned}
& \sigma\left(\mathrm{e}^{+} \mathrm{e}^{-} \rightarrow \mathrm{e}^{+} \mathrm{e}^{-} \mathrm{b} \overline{\mathrm{b} X}\right)_{\text {electrons }}^{\text {total }} \\
& \quad=12.6 \pm 2.4 \pm 2.3 \mathrm{pb}, \\
& \sigma\left(\mathrm{e}^{+} \mathrm{e}^{-} \rightarrow \mathrm{e}^{+} \mathrm{e}^{-} \mathrm{b} \overline{\mathrm{b} X}\right)_{\text {muons }}^{\text {total }}=13.0 \pm 2.4 \pm 2.3 \mathrm{pb},
\end{aligned}
$$

where the first uncertainty is statistical and the second systematic. These results are in perfect agreement with each other and their combination gives

$\sigma\left(\mathrm{e}^{+} \mathrm{e}^{-} \rightarrow \mathrm{e}^{+} \mathrm{e}^{-} \mathrm{b} \overline{\mathrm{b} X}\right)^{\text {total }}=12.8 \pm 1.7 \pm 2.3 \mathrm{pb}$,

where, again, the first uncertainty is statistical and the second systematic. This result is in agreement with our previous measurement performed with just a subset of the data investigated here [8] and has an improved precision.

As a cross check, the values of $N_{\mathrm{c} \overline{\mathrm{c}}}$ found by the fit are used to extract the total cross section for the production of open charm at the luminosity-averaged centre-of-mass energy $\langle\sqrt{s}\rangle=198 \mathrm{GeV}$ as

$$
\begin{aligned}
& \sigma\left(\mathrm{e}^{+} \mathrm{e}^{-} \rightarrow \mathrm{e}^{+} \mathrm{e}^{-} \mathrm{c} \overline{\mathrm{C} X}\right)_{\text {electrons }} \\
& =(10.4 \pm 1.8) \times 10^{2} \mathrm{pb}, \\
& \sigma\left(\mathrm{e}^{+} \mathrm{e}^{-} \rightarrow \mathrm{e}^{+} \mathrm{e}^{-} \mathrm{c} \overline{\mathrm{c} X}\right)_{\text {muons }}=(9.8 \pm 1.6) \times 10^{2} \mathrm{pb},
\end{aligned}
$$

where uncertainties are statistical. These values agree well, and their average

$\sigma\left(\mathrm{e}^{+} \mathrm{e}^{-} \rightarrow \mathrm{e}^{+} \mathrm{e}^{-} \mathrm{c} \overline{\mathrm{c} X}\right)=(10.0 \pm 1.2) \times 10^{2} \mathrm{pb}$

agrees with the dedicated measurement of Ref. [8], $\sigma\left(\mathrm{e}^{+} \mathrm{e}^{-} \rightarrow \mathrm{e}^{+} \mathrm{e}^{-} \mathrm{c} \overline{\mathrm{c} X}\right)=(10.2 \pm 0.3) \times 10^{2} \mathrm{pb}$ for $\langle\sqrt{s}\rangle=194 \mathrm{GeV}$, where the uncertainties are statistical only.

An additional cross check showed that values of the open-beauty cross section determined with the fit procedure discussed above or with a counting method [8] are compatible. In the latter case experimental criteria were chosen to optimise the charm cross section measurement yielding a result essentially uncorrelated with the b-quark production rate.

The total cross section for open-beauty production is compared in Fig. 5 to NLO QCD calculations [7]. The dashed line corresponds to the direct process while the solid line shows the prediction for the sum of direct and resolved processes. The cross section depends on $m_{\mathrm{b}}$, which is varied between 4.5

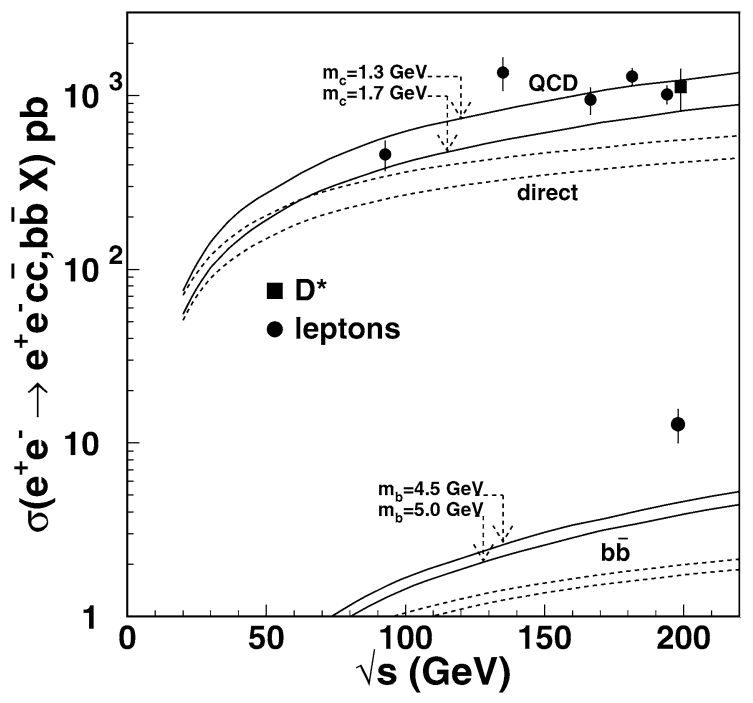

Fig. 5. The open-charm, upper, and open-beauty, lower, production cross sections in photon-photon collisions measured with the L3 detector. Statistical and systematic uncertainties are added in quadrature. The dashed lines correspond to the direct-process contribution and the solid lines represent the NLO QCD prediction for the sum of the direct and single-resolved processes. The effects of a different choice of the values of the quark masses, $m_{\mathrm{c}}$ and $m_{\mathrm{b}}$, are illustrated.

and $5.0 \mathrm{GeV}$. The threshold for open-beauty production is set at $10.6 \mathrm{GeV}$. The theory prediction for the resolved process is calculated with the GRV parton density function [26]. The same results are obtained if the Drees-Grassie parton density function [27] is used. For completeness, Fig. 5 also compares the cross sections for open-charm production measured in Refs. [8, $28]$ with the corresponding predictions.

For $\langle\sqrt{s}\rangle=198 \mathrm{GeV}$ and $m_{\mathrm{b}}=4.75 \mathrm{GeV}$, the cross section expected from NLO QCD is $4.1 \pm 0.6 \mathrm{pb}$, where the uncertainty is dominated by uncertainties on the renormalisation scale and on $m_{\mathrm{b}}$. Our measurement is a factor of three, and three standard deviations, higher than expected. In this respect it is interesting to remark that the prediction of CASCADE, when extrapolated to the full phase space, $3.5 \mathrm{pb}$, agrees with those from NLO QCD [24], and the excess of our data with respect to the expectations is consistent before and after the extrapolation from the fiducial volume to the full phase-space.

In conclusion, all high-energy data collected by L3 at LEP is investigated and the $\mathrm{e}^{+} \mathrm{e}^{-} \rightarrow \mathrm{e}^{+} \mathrm{e}^{-} \mathrm{b} \overline{\mathrm{b} X}$ cross sections are measured within the detector fidu- 
cial volume and found to be in excess with respect to Monte Carlo predictions. The cross sections are extrapolated to the full phase space and found to be in excess with respect to next-to-leading order QCD calculations. This confirms our previous findings based on a subset of the full data-sample.

\section{References}

[1] CDF Collaboration, F. Abe, et al., Phys. Rev. Lett. 71 (1993) 500;

CDF Collaboration, F. Abe, et al., Phys. Rev. D 50 (1994) 4252;

CDF Collaboration, F. Abe, et al., Phys. Rev. Lett. 75 (1995) 1451 ;

CDF Collaboration, D. Acosta, et al., Phys. Rev. D 65 (2002) 052005;

CDF Collaboration, D. Acosta, et al., Phys. Rev. D 66 (2002) 032002;

D0 Collaboration, S. Abachi, et al., Phys. Rev. Lett. 74 (1995) 3548 ;

D0 Collaboration, B. Abbott, et al., Phys. Lett. B 487 (2000) 264;

D0 Collaboration, B. Abbott, et al., Phys. Rev. Lett. 85 (2000) 5068.

[2] P. Nason, S. Dawson, K.R. Ellis, Nucl. Phys. B 303 (1988) 607; P. Nason, S. Dawson, K.R. Ellis, Nucl. Phys. B 327 (1989) 49; W. Beenakker, et al., Nucl. Phys. B 351 (1991) 507;

M. Cacciari, et al., JHEP 0407 (2004) 033, and references therein;

M.L. Mangano, hep-ph/0411020, and references therein; S. Frixione, in: Proceedings of DIS'2004, hep-ph/0408317.

[3] H1 Collaboration, C. Adloff, et al., Phys. Lett. B 467 (1999) 156 ;

H1 Collaboration, C. Adloff, et al., Phys. Lett. B 518 (2001) 331, Erratum;

ZEUS Collaboration, J. Breitweg, et al., Eur. Phys. J. C 18 (2001) 625 .

[4] H1 Collaboration, A. Aktas, et al., hep-ex/0411046; ZEUS Collaboration, S. Chekanov, et al., Phys. Rev. D 70 (2004) 012008.

[5] ZEUS Collaboration, S. Chekanov, et al., Phys. Lett. B 599 (2004) 173.

[6] H1 Collaboration, A. Aktas, et al., hep-ex/0502010.

[7] M. Drees, et al., Phys. Lett. B 306 (1993) 371.

[8] L3 Collaboration, M. Acciarri, et al., Phys. Lett. B 503 (2001) 10.

[9] DELPHI Collaboration, W. Da Silva, in: Proceedings of PHOTON2003, Nucl. Phys. B (Proc. Suppl.) 126 (2004) 185; OPAL Collaboration, A. Csilling, in: Proceedings of PHOTON2000, hep-ex/0010060.
[10] L3 Collaboration, B. Adeva, et al., Nucl. Instrum. Methods A 289 (1990) 35;

L3 Collaboration, O. Adriani, et al., Phys. Rep. 236 (1993) 31; M. Acciarri, et al., Nucl. Instrum. Methods A 351 (1994) 300; M. Chemarin, et al., Nucl. Instrum. Methods A 349 (1994) 345; G. Basti, et al., Nucl. Instrum. Methods A 374 (1996) 293; I.C. Brock, et al., Nucl. Instrum. Methods A 381 (1996) 236; A. Adam, et al., Nucl. Instrum. Methods A 383 (1996) 342.

[11] PYTHIA version 5.722 is used; T. Sjöstrand, preprint CERN-TH/7112/93 (1993), revised in August 1995;

T. Sjöstrand, Comput. Phys. Commun. 82 (1994) 74.

[12] M. Cacciari, et al., Nucl. Phys. B 466 (1996) 173.

[13] G.A. Schuler, T. Sjöstrand, Z. Phys. C 68 (1995) 607; G.A. Schuler, T. Sjöstrand, Phys. Lett. B 376 (1996) 193.

[14] V.M. Budnev, et al., Phys. Rep. 15 (1975) 181.

[15] J.A.M. Vermaseren, Nucl. Phys. B 229 (1983) 347.

[16] KORALZ version 4.02 is used; S. Jadach, B.F.L. Ward, Z. Wạs, Comput. Phys. Commun. 79 (1994) 503.

[17] KORALW version 1.33 is used; S. Jadach, et al., Comput. Phys. Commun. 94 (1996) 216; S. Jadach, et al., Phys. Lett. B 372 (1996) 289.

[18] GEANT version 3.15 is used; R. Brun, et al., preprint CERN DD/EE/84-1 (1984), revised 1987.

[19] H. Fesefeldt, RWTH Aachen Report PITHA 85/2 (1985).

[20] JADE Collaboration, W. Bartel, et al., Z. Phys. C 33 (1986) 23; JADE Collaboration, S. Bethke, et al., Phys. Lett. B 213 (1988) 235.

[21] CASCADE version 1.1 is used; H. Jung, Comput. Phys. Commun. 143 (2002) 100; H. Jung, G.P. Salam, Eur. Phys. J. C 19 (2002) 351.

[22] M. Ciafaloni, Nucl. Phys. B 296 (1988) 49; S. Catani, F. Fiorani, G. Marchesini, Nucl. Phys. B 336 (1990) 18.

[23] H. Jung, Phys. Rev. D 65 (2002) 034015.

[24] H. Jung, M. Hanson, private communication.

[25] S. Eidelman, et al., Phys. Lett. B 592 (2004) 1.

[26] M. Glück, E. Reya, A. Vogt, Phys. Rev. D 46 (1992) 1973.

[27] M. Drees, K. Grassie, Z. Phys. C 28 (1985) 451.

[28] L3 Collaboration, M. Acciarri, et al., Phys. Lett. B 453 (1999) 83 ;

L3 Collaboration, M. Acciarri, et al., Phys. Lett. B 514 (2001) 19 ; L3 Collaboration, M. Acciarri, et al., Phys. Lett. B 536 (2002) 217. 